\title{
Estudo e Testes Conceituais de um Sistema Automatizado para Emenda de Correias Transportadoras
}

\author{
Guilherme Brito Rodrigues *,** Jacó Dias Domingues ** \\ Pablo César da Silva *,** Amauri Coelho Ferraz ${ }^{* *}$ \\ Guilherme Gaigher Netto*,** Jakson Inácio de Andrade**** \\ Philip Von Pritzelwitz ${ }^{* *}$ Gustavo Medeiros Freitas *** \\ * Departamento de Engenharia de Controle e Automação \\ Escola de Minas, Universidade Federal de Ouro Preto \\ Ouro Preto, MG, Brasil, (e-mails: guilherme.brito@aluno.ufop.edu.br, \\ pablo.cesar@aluno.ufop.edu.br,guilherme.netto@aluno.ufop.edu.br). \\ ** Instituto Tecnológico Vale - Mineração, Ouro Preto, MG, Brasil \\ (e-mails: jaco.domingues@itv.org, philip.pritzelwitz@itv.org) \\ *** Departamento de Engenharia Elétrica, Escola de Engenharia, \\ Universidade Federal de Minas Gerais, Belo Horizonte, MG, Brasil \\ (e-mail: gustavomfreitas@ufmg.br) \\ **** Vale S.A. (e-mail: jakson.inacio@vale.com)
}

\begin{abstract}
Belt conveyors play a key role in the bulk materials transportation, offering advantages such as low operating cost and safety application in a wide range of applications, including mining processes. Due to the extensive use, the belts require routine maintenance activities, which can be time consuming and present operational risks. Therefore, this paper proposes the study of a robotized solution to increase the automation degree of the steel cord conveyor belt splicing process. Concept tests are performed to validate some stages of the proposed solution, employing an ABB IRB120 manipulator arm with instrumentation and marking tools to model the belt and make reference indications. The belt dimensions are obtained through optical triangulation, using a green linear laser and a webcam coupled to the robot. The steel cables positionf inside the belt are determined using an inductive sensor. The entire system was implemented using the Robot Operating System (ROS), and the arm command using tools from ROS Industrial, MoveIt!, and the Descartes Path Planning library.

Resumo: Os transportadores de correia (TCs) desempenham papel fundamental no transporte de materiais sólidos, apresentando vantagens como baixo custo de operação e segurança de aplicação em diferentes aplicações,incluindo processos de mineração. Devido ao intenso uso, estas estruturas necessitam de manutenção rotineira. A manutenção dos trechos desgastados da correia demanda um tempo considerável, além de apresentar riscos operacionais aos trabalhadores. Diante disso, este artigo propõe o estudo de uma solução robotizada com a finalidade de aumentar a automatização do processo de emenda de correias. Testes de conceito são realizados para validar etapas da solução proposta, empregando um braço manipulador ABB IRB120 e ferramentas de instrumentação e marcação para modelar a correia e fazer indicações de referência. As dimensões da correia são obtidas através de triangulação óptica, utilizando um laser linear verde mais uma webcam acoplados ao robô. Já a posição dos cabos de aço dentro da correia é determinada utilizando um sensor indutivo. Todo o sistema foi implementado utilizando o Robot Operating System (ROS), e o comando do braço utilizando ferramentas do ROS Industrial, MoveIt! e a biblioteca de planejamento de caminhos Descartes Path Planning.
\end{abstract}

Keywords: Belt conveyors; Automated Splicing Process; Robotic Manipulator; ROS Industrial. Palavras-chaves: Transportadores de Correia; Emenda Automatizada; Manipuladores Robóticos; ROS Industrial. 


\section{Introdução}

Os transportadores de correia (TCs) desempenham papel fundamental no transporte de materiais sólidos, em razão do baixo custo de operação, segurança de aplicação, transporte a longas distâncias e versatilidade em uma diversa gama de aplicações (Association, 2007). Em processos de mineração é extenso o uso de TCs no transporte de grande quantidade de materiais, desde a operação de mina, usina de beneficiamento, pelotização e porto. A Vale, maior empresa de mineração do Brasil, possui aproximadamente 2.000 TCs, totalizando mais de $1.000 \mathrm{~km}$ de correia.

Nos transportadores de longa distância, geralmente são utilizadas correias de cabo de aço que apresentam grande resistência à aplicação de cargas e à ruptura, apresentando melhor desempenho.

Entretanto, em consequência do uso intenso, as correias sofrem desgastes devido a fatores mecânicos como o atrito com material transportado, ao funcionamento inadequado dos rolos, ao excesso de tensão aplicada, entre outros. Estas condições podem impossibilitar a continuidade da operação, ocasionando paradas frequentes do transportador e perda da produção.

Na Figura 1 é exibido o transporte de minério utilizando correia transportadora.

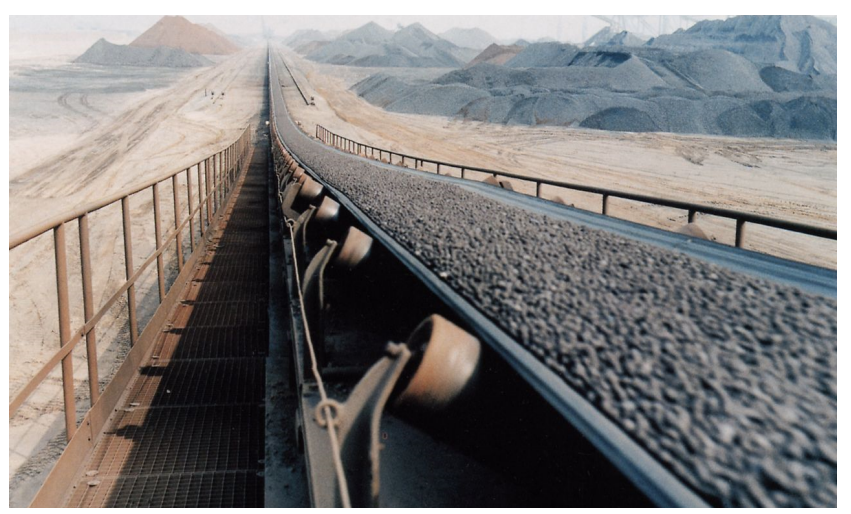

Figura 1. TC utilizado para o transporte de minério.

O processo de manutenção para instalação ou a troca de trechos desgastados das correias transportadoras demanda uma equipe dedicada à realização da atividade. A execução desta atividade se dá por meio de equipamentos e ferramentas na sua maioria manuais. A realização da emenda é um processo praticamente artesanal. Um tempo expressivo é despendido para a realização do procedimento, além de apresentar riscos operacionais e exposição dos trabalhadores a situações ergonomicamente desfavoráveis, como é apresentado na Figura 2.

Diante disso, este artigo propõe analisar uma solução robotizada com a finalidade de aumentar o grau de automatização do processo de emenda de correias com cabo de aço. Os objetivos principais consistem no aumento da segurança dos trabalhadores, otimização da mão de obra utilizada para executar as atividades e redução do tempo para a realização da emenda.

Testes de conceito são realizados para validar etapas da solução proposta, incluindo obter as dimensões da correia e o posicionamento interno dos cabos de aço, e também realizar marcações de pontos de referência na mesma. Para tal, são utilizados um manipulador ABB IRB120, um

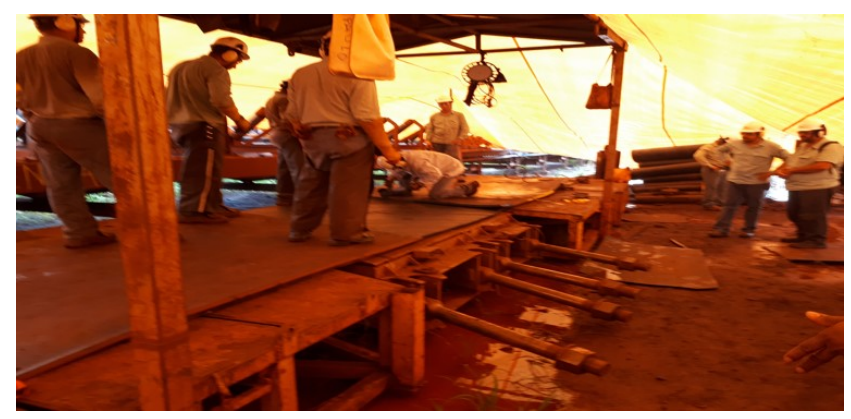

Figura 2. Mobilização de equipe durante processo manual de emenda de correia.

laser linear verde, webcam e sensor indutivo acoplados ao robô. O sistema de validação foi implementado utilizando o Robot Operating System (ROS), e o comando do braço utilizando ferramentas do ROS Industrial, MoveIt! e a biblioteca de planejamento de caminhos Descartes Path Planning.

Na próxima Seção (2) deste artigo serão discutidos os procedimentos para a execução da emenda manual. Em seguida, nas Seções 3 e 4, são apresentadas as soluções propostas para automatização parcial das atividades. $\mathrm{Na}$ Seção 5 são mostrados os resultados obtidos a partir de experimentos realizados em laboratório. Por último, na Seção 6, são apresentadas as conclusões e sugestões de trabalhos futuros.

\section{Emenda Manual}

Atualmente a execução dos procedimentos para emenda de correia transportadora nas áreas operacionais da Vale gasta em média 42 horas de serviço, desde a montagem do espaço de trabalho à vulcanização, utilizando como referência a correia modelo ST 2.500, com $1.800 \mathrm{~mm}$ de largura e 117 cabos de aço internos.

Todas as etapas são realizadas sobre uma estação de trabalho, montada junto à estrutura do transportador e nivelada com o platô da prensa de vulcanização.

Após montagem do espaço de trabalho e sobreposição das pontas a serem emendadas, o próximo passo consiste na marcação do viés (Figura 3a) que corresponde a área de borracha não removível delimitando o comprimento da emenda. Atualmente a marcação é realizada de forma manual por meio de giz e barbante. Na sequência é retirada a cobertura de borracha para exposição dos cabos de aço, fazendo chanfro de $45^{\circ}$ na borda (Figura 3b), utilizando lixadeira e disco de corte.

Com os cabos expostos, o excesso de borracha entre eles é removido individualmente utilizando um filetador ou uma faca olfa, mantendo uma fina camada de borracha (Figura 3c). O material residual na superfície do cabo é desejável para adesão da borracha no procedimento de vulcanização, evitando a necessidade de tratamento do metal.

A etapa seguinte consiste na marcação dos estágios (Figura $3(\mathrm{~d})$ ). Os estágios compreendem às subdivisões onde os cabos serão cortados. O número de estágios pode variar de 1 a 3 passos de corte de acordo com as especificações da correia. No modelo de referência são marcados dois passos de corte onde são estipulados duas dimensões distintas: cabos menores e maiores. Os cabos de aço são cortados com auxílio de um tesourão. 
a)

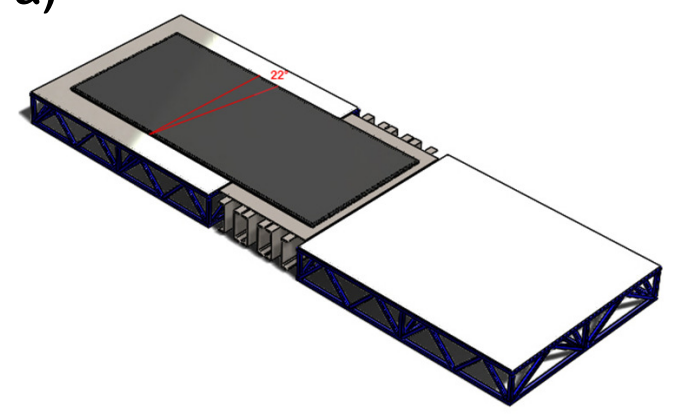

c)

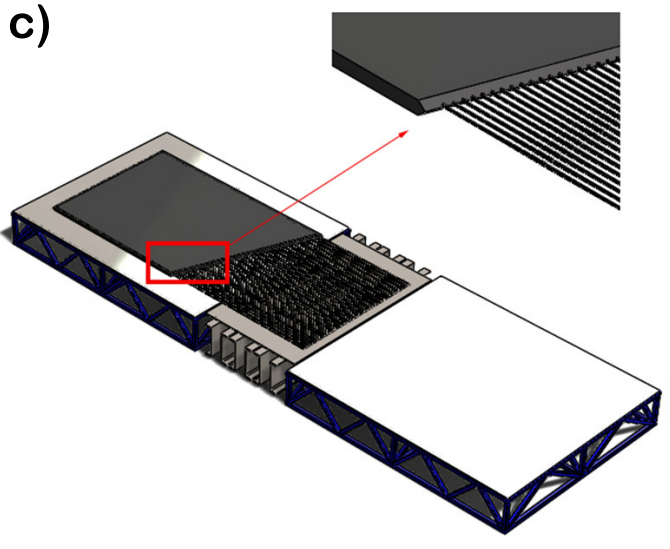

b)
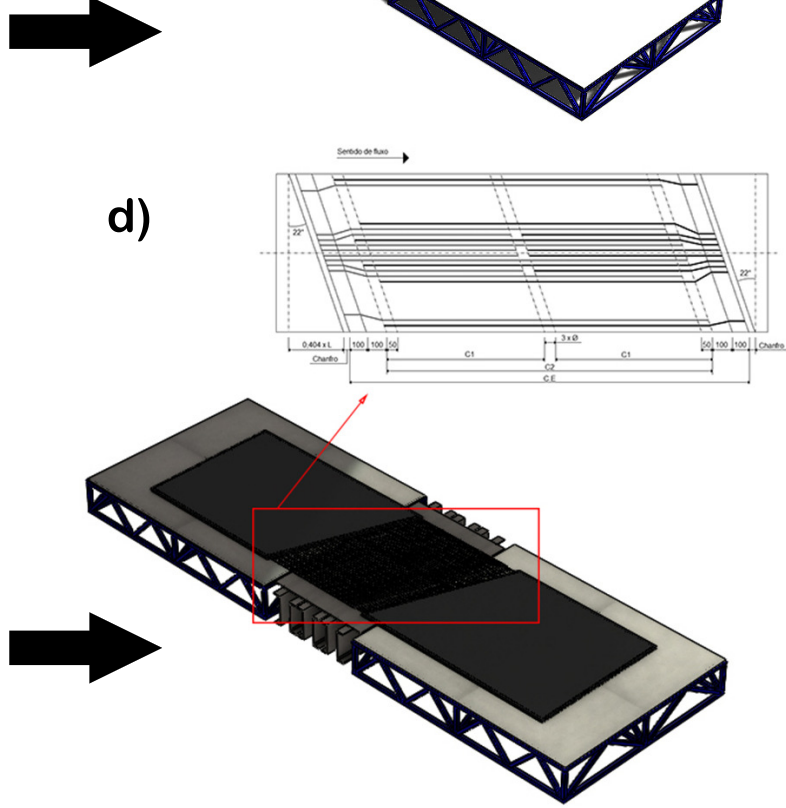

Figura 3. Etapas da emenda de correia: a) Marcação do viés, b) Remoção da cobertura, c) Filetamento dos cabos e d) Marcação dos estágios.

Posteriormente, as duas pontas da correia devem ser alinhadas e fixadas de acordo com o comprimento da emenda sobre o platô da prensa de vulcanização. Os cabos cortados são limpos e após a limpeza, são aplicadas com pincel duas demãos de um adesivo de contato de rápida cristalização.

O próximo passo consiste em posicionar a manta inferior sobre a prensa. As mantas são preparadas antes do serviço de campo com chanfro de $45^{\circ}$ para casar com os chanfros feitos na correia. As mantas devem possuir camadas de borracha de cobertura e ligação.

Os cabos são reposicionados sobre a manta inferior, com cabos maiores na borda com pontas voltadas no sentido contrário da rotação do transportador. A cada dois cabos maiores, são dispostos colinearmente os cabos menores e médios. Os espaços vazios são preenchidos com borracha de ligação.

Em seguida é posicionada a manta superior sobre os cabos e retirado o excesso de borracha da manta, garantindo a cobertura de toda a área da emenda. São posicionadas réguas nas extremidades longitudinais da emenda, com a finalidade de evitar a fuga de material com a pressão e garantir o bom acabamento.

Após o posicionamento da manta superior é realizada a vulcanização da emenda. A vulcanização consiste em um processo químico com a finalidade de melhorar as propriedades físicas da borracha, aumentando a força tênsil e resistência à abrasão e dilatação.

\section{Emenda Automatizada}

Os procedimentos apresentados na seção anterior demonstram o baixo grau de automatização na execução das emendas, com uso de diversas pessoas em atividades de baixa geração de valor por um longo período de tempo. Diante disso este artigo apresenta como proposta automatizar parte das atividades, por meio do emprego de sensores e metodologias para identificação do modelo da correia e realização das marcações dos pontos importantes no processo de emenda com o auxílio de um manipulador robótico. Cabe ressaltar que este é um primeiro passo para o desenvolvimento de um sistema completamente automatizado.

A modelagem da correia é dividida em duas etapas distintas: a modelagem externa, a fim de se obter um modelo geométrico da correia, e a modelagem interna com a finalidade de mapear a disposição dos cabos de aço. A medição das propriedades geométricas é realizada através de técnicas de visão computacional. Para mapeamento da posição dos cabos de aço é proposta a utilização de um sensor indutivo. O modelo da correia é utilizado para planejar a trajetória do braço manipulador a fim de realizar marcações na correia transportadora.

Para realizar a emenda, é necessário conhecer a altura e largura da correia, e também a disposição interna dos 

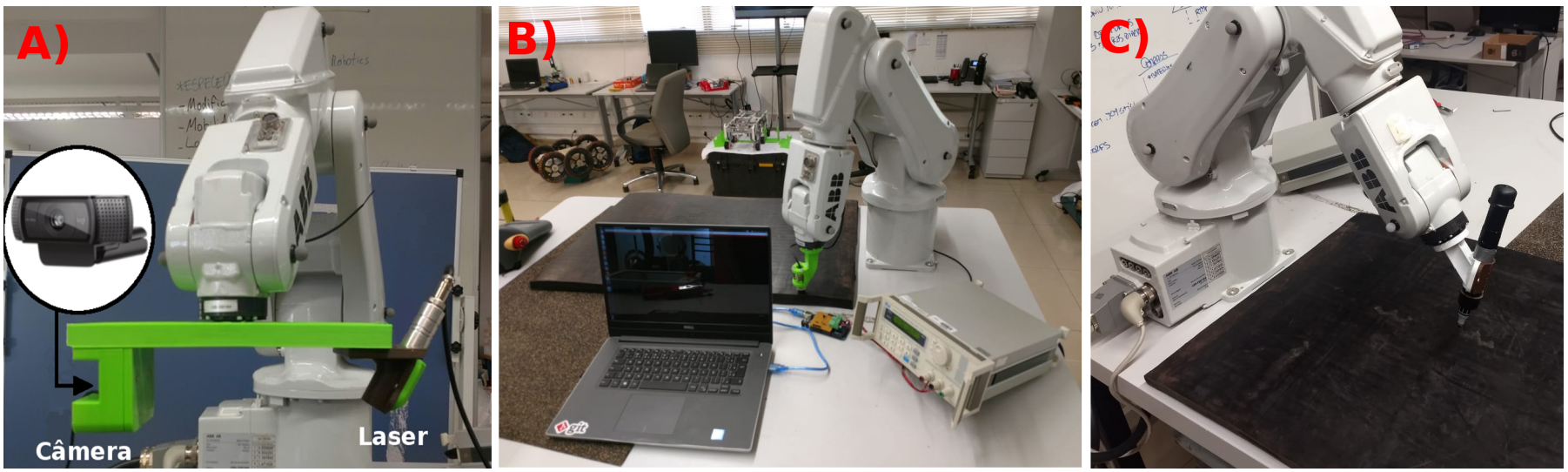

Figura 4. a) Plataforma de encaixe da câmera e laser, b) Execução da modelagem interna da correia, c) Marcação dos pontos de referência.

cabos de aço, conforme apresentado na Figura 5. De acordo com a norma de construção, os cabos devem estar igualmente espaçados com uma distância (d) de $15 \mathrm{~mm}$ e tolerância de $\pm 5 \mathrm{~mm}$ para o modelo de referência.

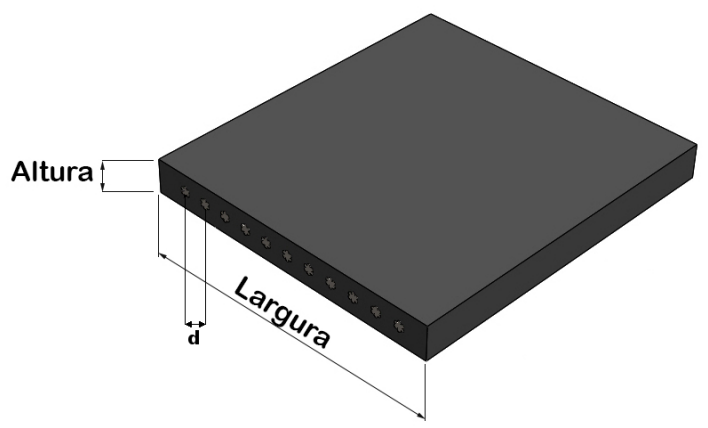

Figura 5. Parâmetros dimensionais da correia.

\subsection{Modelagem Externa}

O processo de criar um modelo geométrico é também chamado de engenharia reversa de forma (Varady et al., 1997), no qual não só uma cópia digital do objeto é criada, mas também são obtidos conhecimentos sobre sua forma. Para obter as medidas da correia é usado um método óptico baseado em triangulação, utilizando laser e câmera.

A triangulação é um método que utiliza as posições conhecidas de uma fonte de luz e de um dispositivo de detecção fotossensível para deduzir a localização de pontos no espaço. Um feixe é projetado, em um ângulo préestabelecido, numa superfície de interesse. Um dispositivo fotossensível, geralmente uma câmera, detecta a reflexão da superfície e usa a triangulação geométrica para calcular posição dos pontos na superfície de interesse em relação ao plano da câmera (Varady et al., 1997). Um esquemático do modelo de triangulação pode ser visto na Figura 6 .

\subsubsection{Método de identificação}

Os dispositivos usados neste artigo para a realização da triangulação foram um laser linear, com comprimento de onda $532 \mathrm{~nm}$ (verde) e potência de $15 \mathrm{~mW}$, como fonte de luz e uma webcam Logitech c920, com resolução de 1080p, como dispositivo fotossensível. Os dispositivos foram montados em uma plataforma impressa em 3D

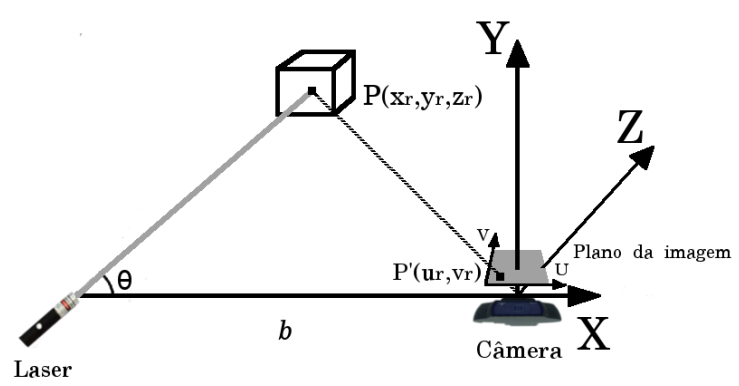

Figura 6. Triangulação geométrica para cálculo de posições espaciais.

(material ABS) com uma distância de $25 \mathrm{~cm}$ e um ângulo de projeção de $60^{\circ}$. A plataforma foi acoplada a um braço robótico para facilitar a modelagem de diferentes seções da correia (Figura 4(a)).

Para medir a altura e largura da correia, um feixe de laser verde é projetado na correia. Uma imagem da cena é então obtida pela câmera. Para medir as posições de cada ponto do feixe é necessário segmentar a imagem, retornando informações apenas do feixe do laser projetado sobre a correia como mostrado na Figura 7.

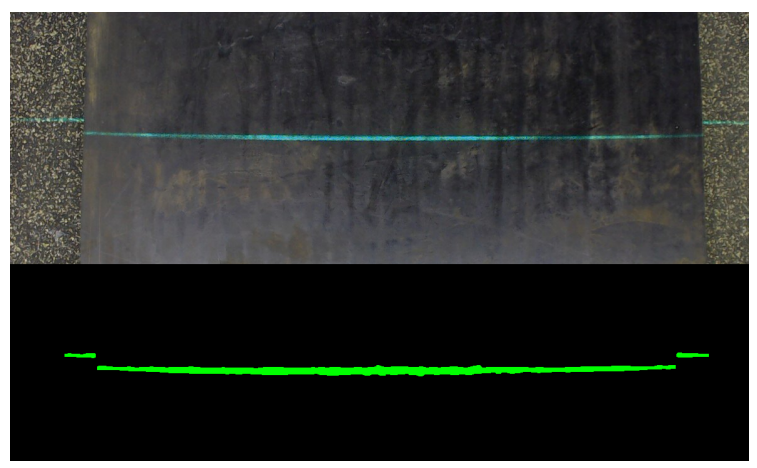

Figura 7. Projeção do laser na correia e segmentação da imagem obtida.

Utilizando as propriedades geométricas da montagem (laser + câmera) é possível identificar a posição de cada ponto do feixe de laser em relação ao centro da câmera através da equação (1): 


$$
\left[\begin{array}{l}
x_{r} \\
y_{r} \\
z_{r}
\end{array}\right]=\frac{b}{f \cot \theta-u_{r}}\left[\begin{array}{c}
u_{r} \\
v_{r} \\
f
\end{array}\right]
$$

onde $\left[x_{r}, y_{r}, z_{r}\right]^{T}$ representa a posição espacial de um ponto do feixe; $b$ a distância entre o laser e a câmera; $\theta$ o ângulo de projeção do laser; $\left[u_{r}, v_{r}\right]^{T}$ a posição (em pixels) do feixe na imagem; e $f$ a distância focal da câmera. Esse procedimento se repete ao longo do comprimento da correia utilizando um movimento linear do braço robótico.

Para obter as medidas da correia utilizando (1) é necessário calibrar a montagem para obter os valores de $b, \theta$ e $f$. Para isso é usado o método de calibração direta similar ao proposto em (Trucco and Verri, 1998). A câmera insere distorções na imagem devido ao seus parâmetros intrínsecos. Portanto, para melhorar a precisão das medidas é necessário também calibrar a câmera utilizado o framework OpenCV (método do "chessboard").

A calibração da montagem (laser + câmera) é feita de acordo com o fluxograma apresentado na Figura 8.

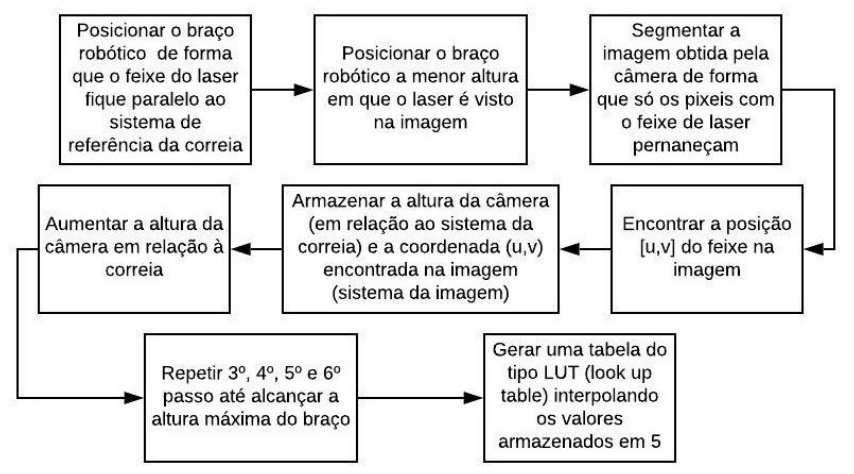

Figura 8. Fluxograma de calibração entre laser e câmera.

Com o tabela LUT pronta pode-se obter a coordenada $Z_{r}$ utilizando as coordenadas do feixe de laser na imagem. As coordenadas $X_{r}, Y_{r}$ podem ser obtidas utilizando as equações de projeção da câmera 2 e 3 e a coordenada $Z_{r}$ calculada anteriormente:

$$
\begin{aligned}
& u_{r}=\frac{f * X_{r}}{Z_{r}} \\
& v_{r}=\frac{f * Y_{r}}{Z_{r}}
\end{aligned}
$$

A altura e largura podem então ser calculadas usando a distância euclidiana dos pontos de interesse, ponto onde o feixe se fragmenta (Figura 7).

\subsection{Modelagem Interna}

A disposição interna dos cabos de aço admite grande tolerância dimensional. Os cabos são paralelamente dispostos, porém não há garantias que a distância de um para o outro seja constante. Nessa situação, um sensor indutivo é utilizado a fim de mapear a posição interna dos cabos de aço ao longo da área de emenda da correia.

Os sensores indutivos são emissores de sinal que detectam elementos metálicos que atravessam o seu campo magnético convertendo em um sinal elétrico inteligível. Esses sensores são constituídos por uma bobina em torno de um núcleo. As características da bobina se alteram na presença de objetos que tenham propriedades magnéticas, pois estes interferem no campo magnético gerado por um oscilador conectado à bobina. Essa variação é sentida e o circuito de disparo interno do sensor altera a tensão de saída fornecendo uma resposta lógica de nível alto ou baixo (Wendling, 2010).

No caso estudado, um sensor com distância de detecção de $10 \mathrm{~mm}$ é acoplado ao manipulador robótico, controlado de forma a identificar os cabos de aço por meio do contato direto com a correia, e realizando trajetórias lineares varrendo toda a malha de aço revestida (Figura 4(b)).

Uma ferramenta foi desenvolvida com a finalidade de acoplar o sensor indutivo ao braço robótico. Para garantir o contato do sensor com a superfície da correia a ferramenta é constituída por molas de compressão com objetivo de proporcionar complacência ao toque.

\subsection{Marcações}

As marcações dos pontos importantes para execução da emenda, como o viés e os estágios de corte, são atualmente realizados de forma manual utilizando giz e barbante a partir de cálculos preliminares.

O viés (Figura 9) é calculado pelo produto da medida da largura da correia considerando a relação trigonométrica $\tan \left(22^{\circ}\right)$.

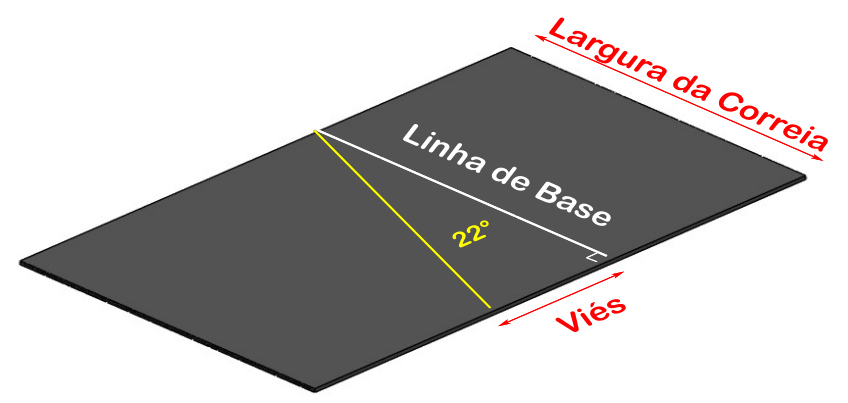

Figura 9. Marcação do viés na correia transportadora.

Utilizando o modelo em três dimensões da correia previamente obtido, é realizado o planejamento de trajetória do robô através de movimentos lineares, de forma a realizar as indicações na correia através de um marcador acoplado ao manipulador (Figura 4(c)).

\section{Planejamento de trajetórias para um Manipulador} Robótico Industrial

O manipulador industrial utilizado neste trabalho, fabricado pela ABB Corporation, consiste em um braço manipulador IRB 120 e um controlador IRC5 Compact, otimizado para pequenos robôs.

O manipulador robótico IRB 120 é o menor braço comercializado pela ABB Corporation, com payload de $3 \mathrm{~kg}$ e alcance horizontal de $580 \mathrm{~mm}$. Este manipulador conta com seis juntas de revolução caracterizando seis graus de liberdade (Robotics, 2017).

A maioria dos robôs industriais utilizam softwares proprietários e controladores nativos, de arquitetura fechada. Segundo Bartolomeu and Almeida (2005), um dos desafios da robótica consiste em integrar as informações vindas de sensores e algoritmos com a finalidade de dar mais flexibilidade à aplicação. Uma maneira de se contornar o problema é implementar o controle dos equipamentos 
à partir de um sistema unificado, onde diversos tipos de algoritmos possam ser aplicados de forma mais simples e centralizada como tratamento de imagens, visão computacional, aquisição de dados dos sensores, entre outras (Torre et al., 2018).

Diante disso, o desenvolvimento desta aplicação é baseada no framework Robot Operating System (ROS) e sua extensão ROS-Industrial, que permite a comunicação entre o ROS e alguns braços industriais fabricados pela ABB, Motoman, Fanuc e Universal Robots (Martin, 2013).

\subsection{Robot Operating System (ROS)}

O ROS é um meta sistema operacional de código aberto para o desenvolvimento de aplicações robóticas. Esse ambiente consiste em uma coleção de ferramentas, bibliotecas e convenções utilizadas para criar sistemas robóticos complexos e robustos para uma grande variedade de plataformas robóticas (Foundation, 2018).

Esse software oferece funcionalidades de um sistema operacional, como abstração de hardware, controle de dispositivos a baixo nível, troca de mensagens entre processos e gerenciamento de pacotes (O'Kane, 2013). Os programas/processos são chamados de nós, podendo ser programados em $\mathrm{C}++$ ou Python. Um sistema robótico é composto geralmente por vários nós, sendo que a estrutura modular do ROS permite a execução destes de forma paralela, se comunicando através de troca de mensagens em estruturas conhecidas como tópicos (Quigley et al., 2009).

Quando comparado a outras plataformas, o ROS se destaca por apresentar um elevado número de componentes e suportar o maior número de robôs (Magyar et al., 2015), além de contar com uma ampla e crescente comunidade de usuários contribuindo para a sua expansão, contribuindo assim para o compartilhamento e reuso de códigos (Staranowicz and Mariottini, 2011).

\subsection{ROS Industrial, MoveIt! e OMPL}

O ROS Industrial consiste em um projeto de código aberto que estende as capacidades do ROS para aplicações industriais (Michieletto et al., 2014).

A interação direta do usuário com o robô acontece por meio do MoveIt! que utiliza algoritmos tidos como estado da arte para manipulação móvel, incorporando os últimos avanços em planejamento de trajetórias, manipulação, percepção $3 \mathrm{D}$, cinemática, controle e navegação. O MoveIt! consiste em uma extensão de fácil utilização para desenvolvimento de aplicações robóticas avançadas, avaliação de projetos e construção de sistemas robóticos integrados para as áreas industriais (Torre et al., 2018).

O pacote do MoveIt! utiliza os planejadores da biblioteca OMPL (Open Motion Planning Library), que implementam diferentes algoritmos de planejamento de trajetória baseados em amostragem.

O objetivo é realizar o controle do braço manipulador e ler os sensores embarcados a fim de modelar e realizar marcações na correia transportadora utilizando um único ambiente de programação, o ROS. As trajetórias do braço são planejadas através do computador pelo software MoveIt!.
O primeiro passo é gerar, a partir do modelo CAD do IRB120, um modelo unificado que fornece todas as informações sobre os elementos de um robô, chamado URDF. Nele estão contidas as informações sobre as dimensões dos elos, tipos de juntas, limites de posições, massa, etc. Com o URDF é criado um pacote de configuração no MoveIt! onde é gerado um grupo de planejamento responsável por realizar os cálculos cinemáticos e planejar as trajetórias do manipulador.

Com o pacote do MoveIt! criado, os pontos gerados para execução da trajetória devem ser enviados para o braço robótico real. No ROS Industrial é disponibilizada uma biblioteca com um socket para comunicação com robôs ABB. O socket é composto por uma arquitetura clienteservidor, e deve ser configurado tanto no computador que será cliente, quanto no controlador IRC5 Compact, que será o servidor.

O socket servidor é um conjunto de seis módulos desenvolvidos utilizando a linguagem RAPID (Linguagem de alto nível para controlar robôs industriais da $\mathrm{ABB}$ ) que devem ser incluídos no controlador IRC5 Compact (Martin 2013).

A camada cliente é responsável por enviar e receber as posições das juntas do controlador, por meio de dois tópicos principais/joint_states que recebe a pose atual do robô e /joint_path_command que envia as posições das juntas para o controlador, após a geração de uma nova trajetória.

Entretanto, os algoritmos de planejamento de trajetória da biblioteca OMPL são focados em aplicações pick and place, onde as poses inicial e final do efetuador do braço são a única entrada para os planejadores de caminho. $\mathrm{Na}$ aplicação em estudo, o manipulador deve seguir um caminho cartesiano pré-definido em que não apenas a posição inicial e final são importantes, mas também o caminho intermediário, permitindo a execução de trajetórias lineares. Neste caso, é proposta a utilização do biblioteca de planejamento de trajetórias Descartes Path Planing.

\subsection{Descartes Path Planning}

Tarefas comuns para manipuladores industriais resultam em redundância cinemática. Como exemplo, nas atividades propostas por este artigo, varredura com sensor indutivo e marcação da correia, é utilizado um braço manipulador de 6 graus de liberdade (GdL) onde a rotação da ferramenta não é completamente definida pelo processo, de forma que as atividades exigem menos GdL que os fornecidos pelo robô.

A fim de executar trajetórias lineares exigidas para execução das atividades, é necessário definir a posição tridimensional do ponto central da ferramenta TCP (do inglês tool center point) mais dois graus de orientação em função do tempo. Para aplicações com essas características é requerido o planejamento de trajetórias chamado Planejamento de trajetórias cartesianas.

Esta seção descreve o planejamento de trajetórias cartesianas contida no pacote Descartes Package, um projeto do ROS Industrial que foi apresentado no ROSCON, um congresso para desenvolvedores ROS, em 2015. De acordo com De Maeyer et al. (2017), que faz uma avaliação do algoritmo em seu trabalho, nenhuma descrição formal do software tinha sido publicada até então. 
No algoritmo, o caminho é dividido em N pontos cartesianos, chamados trajectory points, discretizados o suficiente para garantir as restrições do efetuador. Esses pontos não contém informações de velocidade nem aceleração, apenas o limite máximo de tempo de percurso entre pontos consecutivos. Além disso, o movimento entre dois trajectory points deve ser fisicamente executável pelo robô.

O planejamento de trajetórias pode ser dividido em três fases importantes. Na fase 1, quando necessário, uma dada trajetória é convertida no espaço das juntas. Na fase 2 as soluções criadas na fase anterior são organizadas em um grafo. Finalmente na fase 3 uma busca é realizada nas soluções da fase dois para obtenção da trajetória.

$\mathrm{O}$ algoritmo inicia (fase 1) à partir da lista dos pontos cartesianos que podem ser expressados pelo pose desejada pelo efetuador ou pela posição das juntas. No caso da pose do efetuador, há a necessidade de conversão para posição da juntas de acordo com a cinemática inversa do manipulador antes que a busca no grafo seja iniciada na fase 3 .

Para cada trajectory point amostrado, pode ser atribuído pelo usuário um intervalo de tolerância à pose, definido através de erros aceitáveis para posição e orientação representada por ângulos de Euler.

Como a orientação da ferramenta não corresponde a uma restrição da tarefa, é utilizada uma implementação específica chamada ponto axial-simétrico, que permite rotação livre em torno de um eixo e tolerância zero nos outros; isso torna o manipulador redundante, facilitando a realização da tarefa.

Na fase 2, a posição das juntas são organizadas em nós (vértices) de um grafo. Quando a verificação de colisão é realizada, uma posição em colisão não é adicionada ao grafo. Vários nós podem pertencer ao mesmo trajectory point, como mostrado esquematicamente na Figura 10. O caminho é construído entre os nós de dois trajectory points sucessivos. Antes de adicionar uma aresta entre dois, nós o software verifica se a restrição de tempo é obedecida.

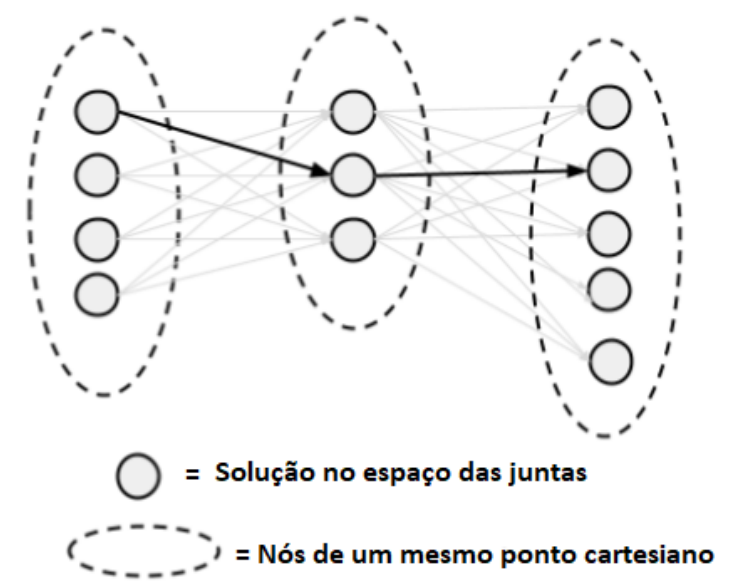

Figura 10. Grafo de planejamento de trajetórias utilizando o Descartes Package.

Fonte: Adaptado de (De Maeyer et al., 2017)

Na fase 3, o grafo direcionado resultante permite o uso de algoritmos de busca em grafos para encontrar o caminho mais curto. O resultado consiste em uma sequência de posições das juntas que, quando seguidas pelo robô, resulta no caminho cartesiano desejado dentro das tolerâncias especificadas; isto ocorre quando o caminho é factível e solucionado pelos algoritmos de solução de cinemática inversa implementados pelo MoveIt!. A sequência de posições no espaço das juntas deve ser facilmente executada pelo controlador do manipulador robótico. $\mathrm{O}$ movimento das juntas pode conter acelerações elevadas e até mesmo provocar colisões em alguns casos; nesta situação é necessário realizar o replanejamento das trajetórias.

Atualmente, o pacote oferece duas implementações de referência de planejadores de trajetória: Dense Planer e Sparse Planer. Ambos aceitam uma sequência de pontos de trajetória arbitrária para entrada, e fornecem como saída de uma sequência de pontos no espaço das juntas.

A versão Sparse Planer permite a execução mais rápida do algoritmo calculando a cinemática inversa apenas para um subconjunto dos trajetory points amostrados. Os pontos intermediários são encontrados pela interpolação dos pontos no espaço das juntas e cinemática direta. Se o erro na trajetória for maior que um limiar pré-definido, um ponto extra é adicionado à sequência dos pontos e o caminho é replanejado.

\section{Testes de Bancada}

A viabilidade das atividades propostas foram realizadas em testes de bancada em laboratório ${ }^{1}$, utilizando uma amostra de correia com revestimento interno de cabo de aço com aproximadamente $57 \mathrm{~cm}$ de largura e $90 \mathrm{~cm}$ de comprimento com 29 cabos dispostos internamente na correia. A posição inicial da correia sobre a bancada é preestabelecida.

O primeiro passo do teste consiste no levantamento da largura e altura ao longo da correia, acoplando a plataforma desenvolvida para fixação do laser e câmera ao manipulador IRB120. O método de identificação é aplicado a alguns pontos, realizando uma varredura da correia. As medidas de altura e largura para cada ponto, associadas à pose do robô, são integrados ao ROS - publicadas em tópicos e salvas em estruturas armazenamento de mensagens chamadas de rosbag.

O próximo passo consiste no mapeamento da disposição interna dos cabos de aço na correia. As trajetórias de varredura do sensor indutivo são planejadas utilizando o algoritmo Descartes Path Planning, assumindo um sistema de coordenadas de referência na borda da correia, e utilizando os dados obtidos de altura e largura na etapa anterior de modelagem. Trajetórias lineares são executadas na superfície da correia permitindo a identificação da posição dos cabos de aço.

A aquisição dos dados fornecidos pelo sensor é realizada por meio da plataforma de prototipagem eletrônica Arduino, que se comunica por meio do protocolo de comunicação Rosserial que funciona sobre a UART do arduino. O firmware executado no dispositivo funciona como um nó completo do ROS, permitindo a publicação dos dados obtidos pelo sensor indutivo.

A cada cabo identificado, a pose do braço robótico IRB120 é salva. A partir do conjunto de posições do manipulador em relação ao sistema de coordenadas de referência, é gerado o modelo interno da correia.

1 Os vídeos demonstrativos dos testes de bancada podem ser encontrados em https://youtu.be/lGI3rAFjRWc. 
O modelo bem definido possibilita o planejamento do caminho para marcação dos pontos importantes no procedimento de emenda. Nos testes de bancada, as marcações de viés e estágios de corte são realizadas por uma adaptação de uma ferramenta de marcação que consiste em um pincel em um tubo com molas, oferecendo complacência ao toque com a superfície da correia. Para execução das marcações, assumindo a largura encontrada da correia e o sistema de coordenadas de referência, o planejamento de trajetória é realizado pelo algoritmo Descartes Path Planning.

\section{Conclusões e Trabalhos Futuros}

Considerando as dificuldades relacionadas ao processo de emenda manual de correias transportadoras, este artigo apresentou uma proposta para a automatização parcial do processo empregando um braço manipulador robótico com diferentes instrumentos acoplados.

Algumas etapas do processo foram verificadas de forma experimental, incluindo a obtenção do modelo da correia e realização das marcações dos pontos preliminares no procedimento de emenda. A automação destas etapas já resultariam num ganho de tempo considerável na realização das atividades iniciais do processo, e correspondem aos primeiros passos necessários para a automatização dos demais procedimentos.

Com o modelo obtido, o próximo passo consiste no acoplamento de outras ferramentas ao braço manipulador para que as atividades de desbaste da borracha, limpeza dos cabos de aço e aplicação do adesivo possam ser realizadas de forma automática, otimizando o processo e garantindo um padrão de qualidade nas emendas executadas.

Entre os benefícios da solução, destaca-se a redução da exposição do trabalhador aos riscos inerentes à atividade de emenda, diminuindo a quantidade de homem-hora para realização dos procedimentos. Esta mão de obra poderá ser alocada para execução de outras tarefas, aumentando dessa forma a produtividade do efetivo.

Apesar da solução se apresentar inviável em alguns casos para aplicação em campo, devido à interferência com outros equipamentos e dificuldade de movimentação, também pode ser utilizada na preparação emenda. Preparação de emenda, normalmente, são realizadas em locais com acesso facilitado, antes da instalação dessa correia sobre o transportador.

\section{Agradecimentos}

Os autores gostariam de agradecer à equipe do Laboratório de Controle, Robótica e Instrumentação do Instituto Tecnológico Vale. O presente trabalho foi realizado com apoio da Coordenação de Aperfeiçoamento de Pessoal de Nível Superior, Brasil (CAPES), Código de Financiamento 001; do Conselho Nacional de Desenvolvimento Científico e Tecnológico (CNPq); da Fundação de Amparo à Pesquisa do Estado de Minas Gerais (FAPEMIG); e da Vale S.A.

\section{Referências}

Association, C.E.M. (2007). Belt Conveyors for Bulk Materials. Conveyor Equipment Manufacturers Association and Conveyor Equipment Manufacturers Association. Engineering Conference.
Bartolomeu, P., L.L.S.L.N.P.A. and Almeida, L. (2005). Integração de informação na equipa de futebol robótico cambada. Electrónica e Telecomunicações.

De Maeyer, J., Moyaers, B., and Demeester, E. (2017). Cartesian path planning for arc welding robots: Evaluation of the descartes algorithm. In 2017 22nd IEEE International Conference on Emerging Technologies and Factory Automation (ETFA), 1-8. doi:10.1109/ETFA. 2017.8247616.

Foundation, O.S.R. (2018). Ros - powering the world's robots. URL http://www.ros.org/.

Magyar, G., Sinčák, P., and Krizsán, Z. (2015). Comparison study of robotic middleware for robotic applications. In Emergent Trends in Robotics and Intelligent Systems, 121-128. Springer.

Martin, J.M. (2013). Trajectory planning for the irb120 robotic arm using ros.

Michieletto, S., Tosello, E., Romanelli, F., Ferrara, V., and Menegatti, E. (2014). Ros-i interface for comau robots. In D. Brugali, J.F. Broenink, T. Kroeger, and B.A. MacDonald (eds.), Simulation, Modeling, and Programming for Autonomous Robots, 243-254. Springer International Publishing.

O'Kane, J.M. (2013). A gentle introduction to ROS. doi: 8-25-2014.

Quigley, M., Conley, K., Gerkey, B., Faust, J., Foote, T., Leibs, J., Wheeler, R., and Ng, A.Y. (2009). Ros: an open-source robot operating system. In ICRA workshop on open source software, volume 3,5 . Kobe, Japan.

Robotics, A. (2017). IRB 120 industrial robot. ABB Corporation.

Staranowicz, A. and Mariottini, G.L. (2011). A survey and comparison of commercial and open-source robotic simulator software. In Proceedings of the 4th International Conference on PErvasive Technologies Related to Assistive Environments, PETRA '11, 56:1-56:8. ACM, New York, NY, USA. doi:10.1145/2141622.2141689.

Torre, M.P., Gabriel, C.G., Queiroz, S.S., de Oliveira Dias, M.S., Souza, M.J.F., and Freitas, G.M. (2018). Desenvolvimento de um sistema para integração de células robóticas industriais com foco na indústria 4.0. Congresso Brasileiro de Automática.

Trucco, E. and Verri, A. (1998). Introductory techniques for 3-D computer vision, volume 201. Prentice Hall Englewood Cliffs.

Varady, T., Martin, R.R., and Cox, J. (1997). Reverse engineering of geometric models - an introduction. Computer-aided design, 29(4), 255-268.

Wendling, M. (2010). Sensores. Universidade Estadual Paulista. São Paulo, 2010, 20. 